\title{
Los mecanismos de la valoración \\ en la construcción del discurso \\ en el aula universitaria
}

$$
\begin{array}{l|l}
\text { Ensayos } & \begin{array}{l}
\text { Revista Colombiana } \\
\text { de Educación, N. } 66 . \\
\text { Primer semestre de 2014, } \\
\text { Bogotá, Colombia. }
\end{array}
\end{array}
$$

\section{//The mechanisms of assessment \\ in the construction of speech in \\ the university classroom}

\section{//Os mecanismos da valoração \\ na construção do discurso na \\ sala de aula universitária}

\section{Mireya Cisneros Estupiñán* \\ Clarena Muñoz Dagua**}

\begin{abstract}
Profesora de la Universidad Tecnológica de Pereira. Correo electrónico: mireyace@gmail.com
Profesora de la Universidad Colegio Mayor de Cundinamarca, Bogotá, Colombia. Correo electrónico: clargui@yahoo.es - clarenamunoz@unicolmayor.edu.co
\end{abstract}

\section{Resumen}

Este artículo se centra en el estudio de la oralidad en el aula universitaria a partir de los fundamentos de la lingüística sistémico funcional (Halliday, 1994). Así, con las tecnologías de la palabra de Ong (1993), analizamos las posibilidades que brindan las prácticas de comunicación oral para la construcción del discurso propio del estudiante universitario. Sobre la base de la lingüística sistémico funcional y el sistema de modo, específico de la función interpersonal del lenguaje, y los referentes de investigaciones anteriores (Muñoz Dagua, 2009; Muñoz Dagua, 2010a; Cisneros-Estupiñán, Rojas y Olave, 2005), justificamos la utilización de la teoría de la valoración (Martin, 2000) relacionada con la evaluación de los recursos léxico-gramaticales de actitud, compromiso y gradación en contextos específicos, como procedimiento metodológico para descubrir los problemas en las interacciones orales en el aula y proponer mecanismos tendientes a mejorar las prácticas discursivas académicas.

\section{Abstract}

This paper focuses on the study of oral speech in the university classroom, using the principles of Systemic Functional Linguistics (Halliday, 1994). Following on Walter Ong (1993), we discuss on the possibilities offered by oral communication practices to guide the construction of university student's own speech. Based on Systemic Functional Linguistics and the Mood System, specific to the interpersonal function of language, and using evidence from previous researches, (Muñoz Dagua, 2009; Muñoz Dagua, 2010; Cisneros-Estupiñán, Rojas y Olave, 2005), we justify the use of the Appraisal Theory (Martin, 2000). This theory is related to the evaluation of grammatical lexical resources, attitude, commitment and gradation in specific contexts as a methodological procedure to identify problems in classroom oral interactions, and suggest mechanisms to improve academic discursive practices.

\section{Resumo}

Este artigo tem foco no estudo da oralidade na sala de aula universitária, partindo dos fundamentos da linguística sistêmico-funcional (Halliday, 1994). Assim, com as tecnologias da palavra de Ong (1993), analisamos as possibilidades que dão as práticas de comunicação oral para a construção do discurso

\section{Palabras Clave}

Oralidad, discurso, lingüística sistémico funcional, teoría de la valoración, educación superior, modo.

\section{Keywords}

Oral, speech, systemic functional linguistics, appraisal tehory, university student higher education, mood.

\section{Palavras chave}

Oralidade, discurso, linguística sistêmico-funcional, teoria da valoração, educação superior, modo. 
próprio do estudante universitário. Baseados na linguística sistêmico-funcional e no sistema de modo, específico da função interpessoal da linguagem, e nos referentes de pesquisas anteriores (Muñoz Dagua, 2009; Muñoz Dagua, 2010a; Cisneros-Estupiñán, Rojas y Olave, 2005), justificamos o uso da teoria da valoração (Martin, 2000) relacionada com a avaliação dos recursos léxico-gramaticais de atitude, compromisso e gradação em contextos específicos, como procedimento metodológico para descobrir os problemas nas interações orais na sala de aula e propor mecanismos que ajudem à melhora das práticas discursivas académicas.

Aunque la oralidad ha sido valorada como una de las habilidades comunicativas más sencillas, rutinarias y cotidianas del ser humano, esta es una actividad decisiva en el trabajo de los estudiantes universitarios porque mediante ella se expresa, amplía, confronta, reelabora y desarrolla el pensamiento cognitivo.

En el aula universitaria, las destrezas y habilidades para la expresión oral deben ocupar un lugar importante, debido a que los universitarios presentan a lo largo de sus estudios serias dificultades al momento de emitir de manera verbal textos y expresarse con fluidez a la hora de atender exposiciones, disertaciones, participaciones y disentir en intervenciones académicas. Dichas problemáticas se expresan en la transmisión incoherente y desorganizada de mensajes, el uso desmedido de barbarismos (redundancias, pleonasmos, reiteraciones, retruécanos, muletillas, entre otras); las deficiencias estructurales de los discursos, las dificultades para acompañar la expresión verbal con lenguajes gestuales y corporales adecuados, las falencias cognitivas y semánticas a la hora de puntualizar precisiones y conceptos, hacer una pregunta e intervenir en el acto pedagógico, entre otras (Muñoz Dagua y Asqueta, 2007; Cisneros-Estupiñán, 2012). Por ello, consideramos que la oralidad en el aula es una competencia esencial que debe ser privilegiada en la formación de los futuros profesionales, quienes requieren desarrollar habilidades comunicativas mediante estrategias didácticas eficientes y productivas que fortalezcan la práctica discursiva adecuada en los espacios académicos (Muñoz Dagua, Andrade y Cisneros-Estupiñán, 2011).

A nuestro juicio, principalmente, son tres los factores causantes de las deficiencias en el manejo de la oralidad en el aula universitaria: uno, en el ámbito educativo, con frecuencia se sugiere que el tema de la oralidad es un problema pertinente a los niveles de primaria y secundaria porque se cree que un estudiante, cuando ingresa a la educación superior, llega con las competencias comunicativas acordes con las necesidades académicas del ámbito uni- 
versitario, pero la realidad evidencia lo contrario. Dos, en los últimos años, los esfuerzos, tanto en la universidad como en los niveles educativos anteriores, se están centrando en cualificar las habilidades lectoras y escritoras y se está descuidando tanto desde las prácticas como desde la investigación- la importancia de la oralidad. Tres, dado que la oralidad "es natural, consustancial al ser humano y constitutiva de la persona como miembro de una especie" (Calsamiglia y Tusón, 1999, p. 27), se considera que no es necesario el énfasis desde las prácticas pedagógicas en el aula, de allí que se ha venido considerando como función prioritaria de la escuela básica la enseñanza de la lectura y de la escritura (Avendaño, 2006, p. 16), y en la universidad se continúa con esta consideración ${ }^{1}$. Sin embargo, es a la universidad a quien corresponde entregar a la sociedad un ser formado para desempeñarse como un profesional que no solo sea buen escritor y buen lector, sino también buen hablante y buen oyente en el contexto de su disciplina.

\footnotetext{
En los currículos de diferentes universidades, se encuentra que en carreras de Derecho y Ciencias Políticas aparece una asignatura de retórica, la cual se dedica más a enseñar aspectos teóricos que aspectos prácticos que puedan ayudar a mejorar la oralidad de los estudiantes. En esta y otras carreras también aparecen asignaturas como "Expresión oral y escrita" o "Comunicación oral y escrita" en las que se dan algunas herramientas de tipo formal para hablar en público. También se encuentran las exposiciones en clase como actividades para evaluar saberes disciplinares sin que efectivamente se trabaje en la cualificación del manejo de la comunicación oral en el aula desde una perspectiva discursiva del lenguaje.
}

Desde esta perspectiva, en el marco de la lingüística sistémico funcional, se ha planteado una investigación de carácter exploratorio $^{2}$ que retoma el modelo de análisis de la función interpersonal, planteada por la lingüística sistémico funcional, específicamente la teoría de la valoración (Martin, 2000), para analizar e interpretar las manifestaciones orales que se dan en el aula de clase universitaria con el fin de evaluarlas, detectar dificultades y proponer estrategias para mejorar los procesos discursivos.

Hemos iniciado nuestra exploración en veinte grabaciones realizadas a exposiciones de estudiantes de los programas de pregrado de la Universidad Colegio Mayor de Cundinamarca y de la Universidad Tecnológica de Pereira. El artículo base, al cual se refieren los estudiantes en sus respectivas presentaciones, es el "Ajedrez persa" (1998), texto que explica la importancia de la notación exponencial y, en general, del conocimiento cuantitativo para conocer y comprender las relaciones de los fenómenos naturales y dar explicación a los pro-

2 Este artículo es un avance de la exploración teórica dentro del desarrollo del proyecto de investigación: "La oralidad en el aula universitaria. Una propuesta didáctica discursiva", que actualmente se adelanta entre la Universidad Colegio Mayor de Cundinamarca (UCMC) y la Universidad Tecnológica de Pereira (UTP).

3 Segundo ensayo del libro Miles de Millones, en que Carl Sagan, en 19 capítulos, desarrolla temas que van desde la importancia de la precisión en los números hasta problemas actuales como el calentamiento global. 
blemas de la ciencia y la sociedad. Esta temática, de interés en las distintas carreras universitarias, porque se relaciona con la inclusión de la perspectiva cuantitativa para la formación del espíritu científico e investigativo, fue clave para la selección del texto.

En el presente artículo exponemos los criterios que hemos considerado para determinar la oralidad en el aula como una competencia esencial que puede coadyuvar con los requerimientos de estudio en forma eficiente y productiva y hacer del acto comunicativo en aula un recurso didáctico que fortalezca la práctica discursiva adecuada en la universidad.

En el orden expositivo, primero se presentan algunos fundamentos acerca de la importancia de la oralidad en el desarrollo cognitivo del ser humano con base en las reflexiones de Ong (1993). En segundo lugar, se determinan las posibilidades que brindan las prácticas de comunicación oral en el aula de clase para la construcción del discurso propio del estudiante a partir de las conclusiones de investigaciones que reivindican las estrategias didácticas de la oralidad en los currículos de las diferentes carreras. En tercer lugar, se presentan argumentos para la utilización de la teoría de la valoración (Martin, 2000) una extensión de los estudios del sistema de modo, específico de la función interpersonal del lenguaje, de la lingüística sistémico funcional, como un procedimiento metodológico oportuno para descubrir los problemas que se presentan en las interacciones orales en el aula de clase y proponer mecanismos tendientes a mejorar las prácticas discursivas. Por último, se apuntan algunas reflexiones para el estudio práctico de las interacciones orales en el aula.

\section{Oralidad y desarrollo cognitivo}

Walter Ong, uno de los más reconocidos integrantes de la escuela de Toronto, en su libro Oralidad y escritura. Tecnologías de la palabra (1993), al explicar el pasaje de la oralidad primaria a la escritura, advierte que los cambios que experimenta la comunicación humana no operan de manera radical, sino mediante procesos que implican la convivencia entre las prácticas que se están superando y las formas que emergen. Para el caso del pasaje de la oralidad primaria a la escritura -la primera de las tecnologías de la palabra- el proceso, según lo explica Ong, fue tan lento que ha sido posible encontrar huellas de la oralidad primitiva durante muchos siglos después de inventada, e incluso consolidada, la escritura. 
Las tecnologías de la palabra y de la comunicación han evolucionado históricamente, de modo que sus cambios han repercutido de manera determinante en las transformaciones mentales y culturales del ser humano. Para Ong (1993, p. 11), el pensamiento y la expresión no son esenciales a la naturaleza humana, sino que poseen características que dependen de los recursos tecnológicos que han sido puestos a disposición de la conciencia. En este contexto, diferencia la oralidad primaria, propia de las culturas que no conocen la escritura o la impresión, y la oralidad secundaria, que correspondería a la cultura actual.

Según el autor, los rasgos de la oralidad primaria se revelan en la preeminencia del estilo aditivo, la cristalización de expresiones iterativas, la frecuencia del recurso de la repetición, la verbosidad, la preferencia por formas tradicionales, la cercanía con el contexto humano inmediato, la tendencia agonística, la identificación comunitaria con el saber, la búsqueda de la autorregulación y el pensamiento situacional. Precisamente, ese mundo oral sonoro, vitalista, totalizador, comunitario- es modificado de manera profunda por la irrupción de la escritura. Esta reduce el sonido dinámico al espacio inmóvil, separa las palabras del presente vivo y no es natural como la oralidad, puesto que se rige por reglas creadas conscientemente.

En el caso de las culturas orales, el almacenamiento del cono- cimiento adquirido dependía de la repetición, por eso estos pueblos poseían patrones de pensamiento formularios y fijos que eran esenciales para la sabiduría y una administración eficaz. Con la creación de la escritura, lentamente, las fórmulas mnemotécnicas perdieron importancia, y cuando la escritura estuvo completamente interiorizada el texto escrito cubrió, con otras formas, las funciones de almacenamiento y difusión de la información.

Así, el texto escrito, apartado del mundo vital humano vivo, visualmente estable, perdurable, determinó la liberación de la mente para el pensamiento más abstracto y original, a la vez que favoreció el análisis, la precisión y un mayor grado de formalidad. Al cambiar la producción y acopio de conocimientos de manera completa, la propia mente también se transforma, dice Ong

Mediante la separación del conocedor y lo conocido, la escritura posibilita una introspección cada vez más articulada, lo cual abre la psique como nunca antes, no solo frente al mundo objetivo externo (bastante distinto de ella misma), sino también ante el yo interior, al cual se contrapone el mundo objetivo. (1993, p.106)

Hoy, cuando la oralidad actúa paralelamente con otras tecnologías de información y comunicación que van más allá de la escritura y el 
libro, la necesidad de volver la mirada a la, de acuerdo con Ong, oralidad secundaria ${ }^{4}$ es apremiante. Esta fue y sigue siendo fundamental en el desarrollo del pensamiento $y$, a nuestro modo de ver, es en presente continuo una herramienta imprescindible en la construcción del discurso del futuro profesional.

De hecho, aunque las nuevas generaciones conviven con numerosos aparatos electrónicos que facilitan el contacto con otros, es la oralidad, finalmente, la herramienta que, tanto a lo largo de la carrera como en las relaciones interpersonales y en la profesión, marcan el acceso a una comunicación integral, pues la mayoría de las actividades cotidianas se llevan a cabo a través de la oralidad (Cisneros-Estupiñán, Rojas y Olave, 2005). Y en el ámbito académico no es para menos: entrevistas, discusiones, exámenes, exposiciones, presentaciones tienen como común denominador la oralidad. Y aunque el desarrollo de habilidades para la construcción del discurso en el aula pareciera un asunto simple, es un problema complejo, pues se trata de volver a la oralidad como una forma idónea de expresar, recrear, representar el conocimiento en el contexto del aula universitaria.

Ya en la antigüedad, Aristóteles, con su Retórica (2002), señaló el camino para el estudio exhaustivo de la palabra para otros. Cuando escribía que "el habla es la representación de la mente y la escritura la representación de la palabra", el filósofo se enfocaba en la necesidad de trabajar, con el orador, en la palabra precisa, clara y efectiva, alejada de los vicios, que lograra en el interlocutor las reacciones presupuestas en el mensaje.

Más adelante, los trabajos de la semiótica moderna sobre la persuasión mostraron que la habilidad para expresar una idea es tan importante como la idea misma, tanto así que es a través de la palabra como se logra, en muchas ocasiones, cambiar una opinión, una decisión o una postura. Por esta razón, la retórica hoy ocupa un lugar fundamental en la academia, la política, la rama judicial y la publicidad. Prometeos y Epimeteos se ven seducidos en el mundo moderno, proclive al consumo, por el gesto, la imagen, el color, el rictus que acompaña la palabra hecha metáfora, metonimia, prosopopeya y personificación (Asqueta y Muñoz Dagua, 2001 y Muñoz Dagua, 2010b).

4 Para Ong, la oralidad secundaria corresponde a una oralidad que se aleja de la sintaxis del libro y de la escritura para ser gramaticalizada por una sintaxis audiovisual que se inicia con el cine, continúa con la televisión y, en la actualidad, se ha extendido a las maquinitas de juego, a los teléfonos móviles, al computador y la Internet. La narración oral supeditada a la imagen, al ícono, a la publicidad. 
La importancia de la retórica en el sistema educativo antiguo y medieval se refleja en el estudio de la estructura lingüística del discurso con la inventio, la dispositio y la elocutio, y en su configuración en la actividad oral, con la memoria y la actio (Barthes, 1993). Con las primeras tres dimensiones, se trataba no solo de hallar los contenidos básicos del discurso, sino también de organizarlos coherentemente en un todo estructurado para ser expuestos al auditorio con un estilo adecuado $y$, de este modo, prever las apelaciones y conseguir el objetivo planteado: suscitar la provocación, la compasión, el miedo, la confianza, el agradecimiento, la adhesión o la envidia por las virtudes del otro. Por su parte, la memorización del discurso implicaba el estudio de una serie de procedimientos para facilitar el recuerdo, mientras que la actio se dirigía a prestar atención a la modulación de la voz y la concordancia entre los gestos y el contenido del discurso.

Los estudios de la retórica hoy le dan menos importancia a la oralidad. No obstante, las posibilidades que ofrece el trabajo sobre la estructuración del discurso desde y en el aula de clases pueden resultar significativas para la formación de los estudiantes. De allí la necesidad de destacar, recuperar y mejorar la oralidad en los procesos de enseñanzaaprendizaje, pues es en el entramado del discurso en el que producción y recepción se dan simul- táneamente, donde cobra sentido la voz del futuro profesional. Saber decir lo que se conoce, discutir con habilidad, convencer con argumentos sólidos no solo hace creíble el discurso, sino que ayuda a cualificar al estudiante en el aula de clase para su profesión o para desempeñarse en cualquier ámbito laboral y lo estimula a optimar sus relaciones interpersonales.

\section{Estrategias orales en la construcción del discurso}

Es frecuente que el trabajo con la oralidad en el aula se limite a buscar criterios de evaluación homogéneos para regularizar y hacer más atractivas y entendibles las exposiciones y las presentaciones orales en las asignaturas de diferentes carreras. En este sentido, la didáctica de la oralidad en el aula universitaria se orienta hacia la elaboración de instrumentos, test o rejillas diseñados para valorar la exposición de los estudiantes. Quizá habría que volver la mirada hacia los niveles escolares cuando la práctica de la oralidad da posibilidades de enriquecer el propio discurso mediante las acciones de los juegos y las actividades lúdicas, con su carácter transaccional, autorregulado y mediador, que permiten interactuar a través de la narración, la argumentación, la explicación y el diálogo.

Olson y Torrance (1998, p. 42) manifiestan la importancia de vin- 
cular en los estudios de oralidad la trama discursiva que representa el sentido unificador del sonido, la fugacidad de la palabra y los mecanismos de recordación o fijación de lo dicho: corporales, expresivos y rituales. Para estos autores, las claves de la oralidad no se encuentran en los mecanismos usados en el intercambio conversacional, sino en el lenguaje utilizado para almacenar información en la memoria. Dicho lenguaje precisa dos reglas: debe ser rítmico y debe ser narrativizado, es decir, su sintaxis debe describir una acción o una pasión, y no principios ni conceptos. Para dar un ejemplo simple, nunca dirá que la honestidad es el mejor principio, sino que el hombre honesto siempre prospera.

Con lo anterior, la construcción del discurso en el aula requiere la capacidad narrativa tanto de docentes como de estudiantes. Narrar es contar hechos, producirlos y construirlos en el intercambio, haciéndolos fluidos y atractivos para otros. Luego, antes que diseñar rejillas para la evaluación de lo que otro dijo, es apremiante que ambos, docente y estudiante, comprendan que una exposición es un relato que se produce en un tiempo y espacio determinados, con personajes y situaciones específicas. El uso de mecanismos que faciliten la empatía con los otros para esa co-construcción es, por tanto, fundamental. Precisamente, con las prácticas de comunicación oral se configuran procesos de producción de sentido en los cuales el discurso es cercano a los participantes y pone en funcionamiento, con los gestos y la expresión, el diálogo que se da con la emisión y la escucha.

Aquí, el uso de mecanismos léxico-gramaticales cumple una labor esencial para la construcción del discurso y, por ende, el desarrollo de una competencia comunicativa adecuada para cada disciplina se hace más urgente. En otras palabras, en las prácticas discursivas en el aula de clase se requiere, como en la estructuración de todo discurso, el desarrollo de conocimientos y habilidades para el uso apropiado de la pregunta retórica, el énfasis, la reiteración, el amontonamiento de palabras, las metáforas, las analogías, las hipérboles, las parábolas (Muñoz Dagua y Asqueta, 2007), la gradación de las palabras y las antítesis que confrontan personajes, situaciones, hechos, cualidades y objetos para construir el conocimiento.

Aparte de estos recursos, es imprescindible profundizar en la explicación y la valoración de los efectos, reacciones, movimientos, cambios que ejercen los gestos y la expresión sobre 
el auditorio en la construcción del discurso. El habla viene acompañada de una serie de signos que en su conjunto presentan o muestran al orador en su integridad. Por tanto, junto a los mecanismos léxico-gramaticales, es preciso examinar y reconocer los códigos sociales y, en suma, las prácticas de los rituales de la interacción, con sus lógicas, en el contexto del aula de clase.

\section{Las interacciones discursivas en el aula en el marco de la lingüística sistémico funcional (LSF)}

Para el estudio de las prácticas discursivas en el aula universitaria, es pertinente retomar el sistema de modo, específico de la función interpersonal del lenguaje, de la lingüística sistémico funcional (LSF) de Halliday (1994), como un procedimiento metodológico oportuno para descubrir los problemas que se presentan en las interacciones orales y proponer mecanismos tendientes a mejorar las prácticas discursivas. La adopción de este modelo y, específicamente, los desarrollos de la metafunción interpersonal de la LSF, con la teoría de la valoración y sus categorías de actitud, compromiso y gradación (Martin, 2000), se debe a que en la oralidad se da una presencia reiterativa de formas evaluativas que expresan la actitud del orador frente a sus proposiciones y propuestas. Como bien lo afirma Kaplan (2007, p. 84), esta teoría "logra una interesante integración teórica que resalta el carácter interpersonal y dialógico de la comunicación humana", igualmente, "el hecho de que los aspectos contextuales y culturales se pongan de relieve en el modelo y se admita la posibilidad de múltiples lecturas de los significados actitudinales" permite analizar los recursos utilizados por estudiantes y docentes para la construcción de su discurso en el aula con la posibilidad de fortalecer criterios para la formación profesional.

En el marco de la LSF, la teoría de la valoración (White, 2004) se ocupa de describir y explicar los recursos lingüísticos mediante los cuales los hablantes llegan a expresar, negociar y naturalizar determinadas posiciones intersubjetivas que son, en última instancia, ideológicas. Como herramienta para el análisis, la teoría de la valoración proporciona un marco para explorar de qué modo y con qué fines retóricos los hablantes y autores adoptan: a) una postura actitudinal hacia el contenido experiencial de sus enunciados, b) una postura hacia sus interlocutores reales o potenciales y c) una postura hacia la heteroglosia del contexto intertextual en el que operan sus enunciados y textos.

La palabra valoración, en su definición, es una noción de amplio alcance que incluye todos los usos evaluativos del lenguaje, mediante los cuales los hablantes y escritores no solo adoptan posturas de valor 
particulares, sino que, además, negocian dichas posiciones con sus interlocutores reales o potenciales. De acuerdo con Hood y Martin (2005, p. 198), la idea básica de la valoración es reorientar la mirada desde el intercambio de bienes y servicios o de información "a la negociación de sentimientos".

En esta teoría se da especial énfasis a los conceptos de Bajtín (1979) acerca de la dimensión dialógica del discurso. En particular, la noción de heteroglosia, que hace referencia a la multiplicidad de lenguajes que operan en una misma cultura. Para este autor, el lenguaje no es unitario, sino que expresa una coexistencia de ideologías, grupos y hasta épocas contradictorios en una sola expresión. Estos discursos se reflejan en el lenguaje cotidiano, en lo que Bajtín Ilama polifonía, término que hace referencia a la presencia de múltiples voces en nuestras propias expresiones, con las cuales nos relacionamos de acuerdo con la autoridad que otorgamos a cada una de ellas.

En este contexto, todo enunciado es un lugar de intercambio verbal que está orientado hacia alguien. El acto comunicativo es un intercambio de voces que se nombran, se reproducen y se manipulan. Cada una de esas voces discursivas se caracteriza por su particular punto de vista y difiere de las otras en sus contenidos ideológicos y axiológicos. La heteroglosia es una expresión del carácter ideológico del lenguaje y de la estratificación social, de manera que el discurso refleja los conceptos del mundo de las clases sociales. En esta perspectiva, para White (2004) cobra igual importancia la noción de intertextualidad, cada enunciado se construye y reelabora sobre la base de enunciados pasados y está disponible para ser empleado de la misma forma en futuros enunciados.

En esta línea de trabajo, la teoría de la valoración contempla la evaluación de los recursos léxico-gramaticales, de actitud, compromiso y gradación utilizados en los discursos en contextos específicos. El primer sistema de actitud alude a los valores que los hablantes comunican en sus juicios y las respuestas emocionales y afectivas que asocian a los participantes y los procesos. Estos pueden estar relacionados tanto con respuestas emocionales como con sistemas de valores culturalmente determinados. El sistema comprende tres regiones semánticas que cubren lo que tradicionalmente se denomina emoción, ética y estética. En el modelo trazado para el estudio de los tres ámbitos, estos se identifican con los subsistemas de afecto, juicio y apreciación. En nues- 
tro corpus de trabajo, por ejemplo, expresiones como "este texto es un poco enredado pero trataré de explicarlo...", "Sagan era un duro para la astronomía", "vale la pena entender lo del ajedrez", "qué historia tan chévere la del origen del ajedrez..." permiten advertir que el sistema de actitud está presente con sus subsistemas en las intervenciones de los oradores del aula de clase universitaria y que se expresa claramente en los recursos léxico-gramaticales utilizados para introducir sus emociones, caracterizaciones y calificaciones tanto del autor como del propio texto al cual hacen referencia.

El sistema de compromiso incluye los recursos por medio de los cuales la voz textual se posiciona intersubjetivamente. En términos de White (2004, p. 1), el compromiso hace referencia a los recursos para posicionar la voz del hablante en relación con las diversas proposiciones y propuestas comunicadas por un texto; los significados por medio de los cuales los hablantes reconocen o ignoran la diversidad de puntos de vista que ponen en juego sus emisiones y por medio de las cuales negocian un espacio interpersonal para sus propias posiciones dentro de esa diversidad.

El lenguaje posiciona a los hablantes y sus textos dentro de la heterogeneidad de posturas sociales y de concepciones del mundo que operan en cualquier cultura. En consecuencia, el discurso refleja una determinada realidad social o una posición ideológica. Cada significado dentro de un texto o discurso ocurre en un contexto social, donde podrían haberse elegido otros significados alternativos o contrarios. Obtiene su importancia y su significado social por las relaciones de divergencia o convergencia que establece en relación con esos significados alternativos. Las principales opciones en el sistema de compromiso se ubican en dos dimensiones contrapuestas: la monoglosia y la heteroglosia. En este punto, en un primer examen de los discursos del corpus podemos verificar la introducción de la voz ajena (del texto de Sagan) como voz propia como sucede en: "verdaderamente, yo digo, que conocer algo de forma meramente cualitativa es conocerlo de manera vaga...", "me parece que en cualquier carrera, si tenemos conocimiento de lo cuantitativo, estamos comenzando a conocerlo en profundidad y accedemos a su poder y al conocimiento que proporciona...". En estos casos, vale la pena reflexionar, hasta dónde se recrea el conocimiento en las intervenciones orales, cómo construye el estudiante su discurso y qué recursos utiliza para diferenciar su voz de la de los autores que recrea en sus exposiciones.

El tercer sistema, gradación, hace referencia a los valores por medio de los cuales los hablantes gradúan (aumentan o disminuyen) el impacto interpersonal, la fuerza o el volumen de sus emisiones y 
regulan (desdibujan o agudizan) el foco de sus categorizaciones semánticas. Las dos categorías principales de la gradación son la fuerza y el foco. En este caso, el uso de diminutivos es una muestra del uso de estas formas de evaluación en las exposiciones de los estudiantes: "es muy poquito lo que se puede agregar...", "Ios numeritos son precisos...", "las matemáticas son un poquito difíciles...", "excelente el relato del origen del ajedrez, sabía solo un poquito por algo que dijo un amigo en...", "solo unos granitos de trigo pueden aumentar tanto hasta agotar un reino", "este es como un ensayito de Carl Sagan", "el textico es interesante".

En síntesis, al avanzar con un primer análisis de las transcripciones de las exposiciones de los estudiantes, es posible advertir una serie de frases, muchas veces reiterativas, con las cuales realizan juicios, apreciaciones y adoptan posturas frente al acto mismo de exponer ante sus compañeros de estudio. Así, el uso de expresiones como: "a mí me tocó exponer...", "algunos dicen que...", "los estereotipos son como las cosas que uno se imagina", "pero... ¿alguien sabe dónde se inventó el ajedrez?", "y bueno, algunas cosas eran difíciles, pero la profe dijo que...", "lo que me toca exponerles ahora es de Sagan...", tomadas del corpus de estudio, muestran cómo el uso de mecanismos léxico-gramaticales cumple una labor esencial para la construcción del discurso. Pero la tarea de analizar e interpretar el uso adecuado a los contextos de estos mecanismos requiere el concurso de docentes no solo de lenguaje, sino de todos los componentes temáticos de los programas. De hecho, el ejercicio de hacer consciente la diferencia entre el discurso propio y el discurso ajeno precisa de unos mínimos intercambios que se deben generar desde las formas de evaluación, programados para los planes de estudio.

Precisamente, con estos ejemplos queda abierta la necesidad de desarrollar una competencia comunicativa adecuada que permita a los estudiantes unas prácticas discursivas en el aula, fundamentadas en la estructuración adecuada del discurso, el desarroIlo de conocimientos y habilidades para el uso apropiado de la pregunta retórica, el empleo del énfasis y la reiteración como formas de subrayar los elementos centrales, la utilización de recursos como metáforas, analogías, hipérboles, las parábolas (Muñoz Dagua y Asqueta C. 2007), gradación de las palabras y las antítesis para construir un discurso integral e integrado a las necesidades del auditorio: profesores y compañeros de labores académicas. 
Luego, con el primer análisis sobre los enunciados que aparecen en los discursos de los estudiantes surge una serie de preguntas que guía la investigación. El interrogante primario apunta a: ¿de qué manera aprovechar la expresión oral como estrategia didáctica en el aula de clase de los universitarios? Pero la respuesta a esta pregunta implica otros cuestionamientos relacionados con las manifestaciones orales que tienen los estudiantes y, sobre todo, conocer cuál es la práctica que tiene el maestro con el discurso oral de los estudiantes en aula. Desde este enfoque, consideramos imprescindible profundizar en la explicación y la valoración de los efectos, reacciones, movimientos, cambios que ejercen los gestos y la expresión sobre el auditorio en la construcción del discurso. El habla viene acompañada de una serie de signos que, en su conjunto, presentan o muestran al orador en su integridad. Por tanto, junto a los mecanismos léxico-gramaticales, es preciso examinar y reconocer los códigos sociales y, en suma, las prácticas de los rituales de la interacción, con sus lógicas, en el contexto del aula de clase (Muñoz Dagua, 2010c).

En este orden de ideas, la aplicación de las categorías de la teoría de la valoración, como se puede prever, resulta pertinente para reconocer la función que cumple la inscripción de la actitud en la explicación de las temáticas, teniendo en cuenta que esta categoría se relaciona con el acercamiento de los conceptos, por parte del orador, mediante la apelación a los juicios y apreciaciones sobre los objetos y el uso de mecanismos como la analogía, la metáfora, la hipérbole y ciertos mecanismos de acentuación de las palabras.

Asimismo, la adopción de esta metodología en el análisis de los discursos que se presentan en el aula de clases permite mostrar cómo, al realizar evaluaciones con recursos del sistema de compromiso, el emisor establece alianzas con los receptores que comparten su punto de vista, se acerca a ellos y neutraliza, de alguna manera, las diferencias o distancias que ocasiona el contacto entre estudiantes y profesores. El estudio de los valores de la probabilidad, la apariencia, el rumor o la habladuría y otros recursos relacionados con la manifestación de la opinión y el afecto al interlocutor proporcionan un marco para analizar la función que cumple este recurso en el discurso oral.

Un tercer elemento, fundamental en el análisis de la oralidad, es la indagación sobre las formas en que el orador gradúa el contacto con el público y, al mismo tiempo, los mecanismos que adecúa para dar fuerza a sus explicaciones. En efecto, la reiteración de formas evaluativas en los discursos orales, al igual que los gestos, son indicios de cómo se realizan el poder, el contacto y el afecto hacia el interlocutor. 
De este modo, con el concurso de la teoría de la valoración, se pueden analizar los recursos que se eligen en las interacciones en el aula para conseguir la explicación, mantener el contacto con los lectores, negociar un espacio de diálogo en el aula de clases y fortalecer el discurso académico. Consideramos estos elementos fundamentales para postular la competencia comunicativa que se genera con el desarrollo de la oralidad como una herramienta necesaria para la construcción del discurso del profesional en el aula. La comunicación oral es una condición básica de la vida laboral contemporánea. De tal manera que saber hablar, saber intervenir en una reunión, hacer una ponencia, preguntar de manera adecuada, debatir una idea, presentar un argumento, dar una entrevista de trabajo exige una preparación que debe mantenerse durante la vida escolar. En la universidad, el proceso debe facilitar el desarrollo de las habilidades lingüísticas y comunicativas en el mismo acto educativo.

\section{A modo de cierre}

Con este acercamiento a la oralidad, señalamos tres criterios que consideramos fundamentales para el estudio práctico de las interacciones orales en el aula. En primer lugar, la opción por una formación integral del estudiante significa que, con los contenidos de los componentes temáticos, este debe ir, paralelamente, asimilando los instrumentos y habilidades para la construcción de su propio discurso, por tanto, corresponde al docente interesarlo en los mecanismos de su propia comunicación.

La indagación por los recursos de la teoría de la valoración utilizados por estudiantes y docentes en sus intervenciones permiten el acercamiento a los textos orales que se producen en sus contextos de uso y explicar las razones por las cuales resultan más eficaces algunas estrategias de interacción que otras en las dinámicas de enseñanza-aprendizaje. De modo que los estudios sobre la metafunción interpersonal son fundamentales para la descripción y comprensión de la evaluación en los discursos orales y la interpretación de los recursos que utilizan los hablantes para establecer el afecto, el contacto y el poder con los interlocutores.

Acorde con la experiencia docente, el contacto valorativo es esencial para establecer un grado de intimidad en el acto educativo. La posibilidad de evaluar los conceptos que se describen y explican permite tomar un posicionamiento ante los sistemas 
de valores que circulan en la sociedad y acercarse más al interlocutor. Estas valoraciones tienen como base conocimientos que se apoyan, por una parte, en el saber científico $y$, por otra, en las creencias que se comparten con la audiencia.

En segundo lugar, volver sobre la oralidad significa replantear la forma como se construye o reconstruye el conocimiento en el aula. La mirada sobre el conocimiento narrativizado, cercano a oradores y oyentes, esto es, a docentes y estudiantes, señala la conversión del proceso enseñanza-aprendizaje en un espacio ideal para reforzar la confianza entre los actores $y$, así, en un clima cercano entre los protagonistas, corregir los errores, explicar con precisión, exponer con claridad, discutir con argumentos, disentir con evidencias, evaluar con objetividad, cuestionar con rigor, hablar con fluidez y fortalecer los vínculos interpersonales. En este sentido, la contextualización del trabajo en la didáctica crítica y la filosofía del taller (Andrade y Muñoz Dagua, 2004) representan la búsqueda de situaciones de interacción que permitan desarrollar las competencias comunicativas, propositivas, argumentativas e interpretativas del estudiante y asegurar de esta forma su mirada crítica y autónoma frente al contexto escolar, familiar y social.

En tercer lugar, comprendemos que pasar de la idea a la palabra requiere entrar a participar con otros en una dinámica en la que el discurso es aquí y ahora. En esta perspectiva, la oralidad genera autonomía para la construcción de conocimientos propios, pues el participante del acto comunicativo es consciente de lo que dice, abstrae significados no para sí, sino para otros. Luego, esta mirada sobre el discurso que construye con los intercambios en el aula le permite hacer suyos gestos, palabras, rituales que enriquecen su discurso profesional: selecciona el saber que considera útil y valora la información y el conocimiento de acuerdo con las dinámicas de su quehacer individual y el clima organizacional, generado en el aula de clase con la puesta en escena del diálogo, la discusión y la confrontación con los coparticipantes.

\section{Referencias bibliográficas}

Andrade, M., y Muñoz Dagua, C. (2004). El taller crítico: una propuesta de trabajo interactivo. Tabula Rasa (2), 251-262.

Aristóteles. (2002). Retórica. Madrid: Alianza Editorial.

Asqueta, C., y Muñoz Dagua, C. (2001). La fábula del buhonero: semiótica de la estética mercantil. Bogotá: La Tadeo-Uniminuto. Avendaño, F. (2006). Comprensión y producción de textos orales en el aula. En N. Desinano, y F. Avendaño (s. f.), Didáctica de las ciencias del lenguaje. Rosario: Homo Sapiens Ediciones. 
Bajtin, M. (1979). Estética de la creación verbal. México: Siglo XXI. Barthes, R. (1993). La aventura semiológica. En R. Barthes (s. f.), La retórica antigua. Barcelona: Paidós.

Calsamiglia, H. y Tusón, A. (1999). Las cosas del decir: manual de análisis del discurso. Barcelona: Ariel.

Cisneros-Estupiñán, M. (2012). Caracterización de la oralidad en exposiciones de estudiantes universitarios. Pereira: Universidad Tecnológica de Pereira.

Cisneros-Estupiñán, M., Rojas, I. y Olave, G. (2005). Marcas orales en textos escritos por estudiantes que ingresan a la universidad. En L. Ramírez, y A. G. (s. f.), Estudios del discurso en Colombia (pp. 273-294). Medellin: Universidad de Medellin y Aled.

Halliday, M. (1994). An introduction to functional grammar. Londres: Edward Arnold Publisher.

Hood, S. y Martin, J. (2005). Invocación de actitudes: el juego de la gradación, de la valoración en el discurso. Revista Signos, 58(38), 195-220.

Kaplan, N. (2007). La teoría de la valoración: un desarrollo de los estudios sobre la evaluación en el lenguaje. En A. Bolivar (comp.), Análisis del discurso ¿por qué y para qué? Caracas: Universidad Central de Venezuela.

Martin, J. R. (2000). "Beyond exchange: Appraisal systems in English", S. Hunston \& G. Thompson (Eds.): Evaluation in text: Authorial stance and the construction of discourse. Oxford: Oxford University Press, pp. 142-175.

Muñoz Dagua, C y Asqueta, C. (2007). Los estudiantes universitarios y los discursos. La posibilidad de una neogramática. Mediaciones, 7, 27-41.

Muñoz Dagua, C. (2009). Caracterización y funcionalidad de la metáfora interpersonal léxico-gramatical en el discurso de divulgación científica. Un enfoque sistémico funcional. (Tesis de doctorado). Universidad de Buenos Aires, Buenos Aires.

Muñoz Dagua, C. (2010a). El rol de la metáfora léxica en la divulgación. En: Tabula Rasa: Revista de Humanidades de la Universidad Colegio Mayor de Cundinamarca, 213-221.

Muñoz Dagua, C. (2010b). La metáfora interpersonal léxico-gramatical. Un recurso eficaz para la divulgación de la ciencia. Rasal: Revista de la Asociación Argentina de Lingüística, 1 (2), 25-44. 
Muñoz Dagua, C., Andrade, M., y Cisneros-Estupiñan, M. (2011). Estrategias de interacción oral en el aula: una didáctica crítica del discurso educativo. Bogotá: Editorial Magisterio.

Muñoz Dagua, C. (2010c). "Las tecnologías de la palabra y su incidencia en la construcción de una neogramática". Ponencia en II Seminario Internacional de Lectura en la Universidad y I Congreso Nacional de Expresiones de Cultura Escrita en Instituciones de Educación Media, y Superior. Universidad Autónoma de Aguascalientes. Aguascalientes, México.

Olson, D. y Torrance, N. (comp.). (1998). Cultura escrita y oralidad. Barcelona: Gedisa.

Ong, W. (1993). Oralidad y escritura. Tecnologías de la palabra. Buenos Aires: Fondo de Cultura Económica.

Sagan, C. (1998). Miles de millones. Pensamientos de vida y muerte en la antesala del milenio. Barcelona: Ediciones B.S.A.

White, P. (2004). The language of attitude, arguability and interpersonal positioning. Recuperado de http://www.grammatics. com/appraisal/index.html

White, P. (2005). An introductory Course in Appaisal Análisis. http://www.grammatics.com/ appraisalGuide/UnFramed/ IndexOfUFramedGuide.html. 
